\title{
Network Pharmacology-Based Antioxidant Effect Study of Zhi-Zi-Da-Huang Decoction for Alcoholic Liver Disease
}

\author{
Li An ${ }^{1}$ and Fang Feng ${ }^{1,2}$ \\ ${ }^{1}$ Department of Pharmaceutical Analysis, China Pharmaceutical University, Nanjing 210009, China \\ ${ }^{2}$ Key Laboratory of Drug Quality Control and Pharmacovigilance, China Pharmaceutical University, Ministry of Education, \\ Nanjing 210009, China \\ Correspondence should be addressed to Fang Feng; fenglfang1@126.com
}

Received 13 May 2014; Revised 6 August 2014; Accepted 13 August 2014

Academic Editor: Srinivas Nammi

Copyright (C) 2015 L. An and F. Feng. This is an open access article distributed under the Creative Commons Attribution License, which permits unrestricted use, distribution, and reproduction in any medium, provided the original work is properly cited.

\begin{abstract}
Zhi-Zi-Da-Huang decoction (ZZDHD), a classic traditional Chinese medicine (TCM) formula, has been used for centuries to treat alcoholic liver disease. Reliable therapeutics of ZZDHD has also been validated in clinical practice. In this study, molecular docking and network analysis were carried out to explore the antioxidative mechanism of ZZDHD as an effective therapeutic approach to treat alcoholic liver disease. Multiple active compounds of ZZDHD were screened based on four key original enzymes (cytochrome P450 2E1, xanthine oxidase, inducible nitric oxide synthase, and cyclooxygenase-2) involved in ethanol-induced oxidative stress damage. A drug-target network was constructed through network pharmacology analysis, which predicted the relationships of active ingredients to the targets. Some results had been verified by the previous experimental pharmacological studies; meanwhile, it was first reported that xanthine oxidase and eriocitrin, neoeriocitrin, isorhoifolin, and poncirin had interactions. The network pharmacology strategy used provided a forceful tool for searching the mechanism of action of TCM formula and novel bioactive ingredients.
\end{abstract}

\section{Introduction}

Alcoholic liver disease (ALD) is one of the major diseases threatening human health and is also the leading cause of liver-related morbidity and mortality worldwide $[1,2]$. Although no single process or underlying mechanism can account for all the effects of alcohol on an organism or even on one specific organ [3], it has been proven that reactive oxygen species (ROS) and oxidative stress play central roles in the early stage of the disease process [4]. In ethanolinduced liver damage, ethanol can increase the generation of reactive oxygen species (ROS) such as $\mathrm{O}_{2}{ }^{-\bullet}, \mathrm{H}_{2} \mathrm{O}_{2}$, and excessive oxygen free radicals disrupt the balance between the oxidation and antioxidation systems, further causing oxidative damage. Moreover, ROS cause the lipid peroxidation of cellular membranes [5], and lipid peroxidation in turn further aggravates ROS generation [6]. Thus, oxidative stress results from excessive oxygen free radical formation and antioxidant depletion due to the ROS generated by ethanol administration [7].
In early-stage alcoholic liver damage, these two enzymes, cytochrome P450 2E1 (CYP2E1) and xanthine oxidase (XO), have been proven to generate ROS in liver as a consequence of alcohol exposure $[8,9]$. Ethanol mainly increases the activities of CYP2E1 and $\mathrm{XO}$, which can generate excessive oxygen free radicals and lead to lipid peroxidation, further causing oxidative stress damage. Oxidative stress mainly increases the activities of inducible nitric oxide synthase (iNOS) and cyclooxygenase-2 (COX-2), further aggravating lipid peroxidation and oxidative stress $[10,11]$. In ethanolinduced liver damage, four enzymes including CYP2E1, XO, COX-2, and iNOS are the original proteins described as generating ROS or oxidative stress, and they play important roles in the damage due to oxidative stress. Hence, the timely and effective control of high activities of these enzymes, which would eliminate ROS or relieve oxidative stress, is beneficial for the treatment of alcoholic liver injury.

Zhi-Zi-Da-Huang decoction (ZZDHD), a traditional Chinese medicine (TCM) formula, was first described in 
the book of Jin-Kui-Yao-Lue (Synopsis of Golden Chamber). ZZDHD, consisting of four crude herbs: Gardenia jasminoides Ellis (Zhi-Zi), Rheum officinale Baill (Da-Huang), Citrus aurantium L. (Zhi-Shi), and Semen Sojae Preparatum (Dan-Dou-Chi), has been reported to have the ability to treat or alleviate the symptoms of alcoholic jaundice, alcoholic liver disease, and acute hepatitis [12]. Wang et al. [13] reported that ZZDHD had a significant protective effect by reversing biochemical parameters and histopathological changes, and its antioxidant function ameliorated the hepatic injury induced by alcohol. Thirty active components of ZZDHD have also been reported [14]; however, it remains unclear how the active ingredients function as antioxidants.

Network pharmacology, a key technology of system biology, has attracted much attention by researching the molecular mechanisms of TCM formula for complicated diseases [15-17]. Zhang et al. [18] studied an integrative platform of TCM network pharmacology and its application on a herbal formula. Li et al. [19] also determined active compounds and action mechanisms of Ge-Gen-Qin-Lian decoction for treatment of type 2 diabetes by network pharmacology method. Many active chemical compositions of TCM target multiple proteins in the biological network of some disease. Molecular docking is available for modeling interactions between small molecules and proteins. Thus, research of TCM based on network pharmacology, which is a holistic understanding of the molecular mechanisms responsible for the pharmacological effects of herbal medicines $[17,20]$, is well worth exploration.

In this study, a network pharmacology study of ZZDHD was established through molecular docking and network analysis based on thirty identified active components of ZZDHD and four potential targets including cytochrome P450 2E1 (CYP2E1), xanthine oxidase (XO), inducible nitric oxide synthase (iNOS), and cyclooxygenase-2 (COX-2). The study provides a powerful tool for explaining the antioxidant mechanism of TCM formula and discovering novel bioactive ingredients.

\section{Materials and Methods}

2.1. Potential Targets and Ligand Structures Preparation. Reactive oxygen species are the major prooxidant agents in oxidative stress-induced lipid peroxidation, which results in oxidative damage to various types of cell components including lipids, proteins, and DNA [21-23]. The crystal structures of all candidate targets were retrieved from RCSB Protein Data Bank (http://www.pdb.org/), and relevant proteins including CYP2E1 (PDB ID code 3E6I), XO (PDB ID code 3NRZ), COX-2 (PDB ID code 3PGH), and iNOS (PDB ID code 1M8E) were chosen as potential targets of oxidative-stress damage. All proteins were performed using the CHARMm Force Field with the help of the software package Discovery Studio 2.5 (Accelrys, USA). A total of 30 chemical structures of ZZDHD were identified by HPLCPDA-ESI-MS/MS in our early laboratory work (see Figure S1, available online at http://dx.doi.org/10.1155/2014/492470), and all the structures of these compounds were optimized by MMFF94 Force Field in DS 2.5.
2.2. Molecular Docking and Network Building. Molecular docking was conducted with the LibDock protocol based on the CHARMm Force Field in DS2.5. LibDock is considered to balance speed and accuracy and is based on the matching of polar and apolar binding site features of the protein-ligand complex. In the docking procedure, high quality was set for the docking preference parameter, and the best was set for the conformation method. The other parameters were used as the default. In general, the proteinligand docking active site is defined by the location of the original ligand. A compound of ZZDHD was considered to be a potentially active ingredient if the LibDockScore of the compound was higher than the original ligand. Conversely, the compound was not considered if it was not shown at the binding site of the protein-ligand complex. The drugtarget network was then constructed using Cytoscape 3.0.2 software (http://www.cytoscape.org/) based on the top 10 of the molecular docking rank. In the network, nodes stand for compounds and targets, and edges represent the compoundtarget interactions.

\section{Results and Discussion}

3.1. Network Construction of Molecular Docking-Based Pharmacology. The active ingredients of ZZDHD were predicted through molecular docking. To further illuminate the relationship between effective compounds and potential targets, a drug-target network was built through network analysis (Figure 1). Multiple active pharmaceutical ingredients of ZZDHD were found to affect different targets.

The network showed the interactions of $\mathrm{XO}$ with 10 compounds such as hesperetin and naringenin, COX-2 with 10 compounds such as aloe-emodin and hesperetin, and iNOS with 5 compounds such as aloe-emodin and naringenin; CYP2E1 showed interactions with only umbelliferone. Previous laboratory pharmacological studies have provided much information about the chemical compounds screened and the corresponding targets. Park et al. reported [24] that aloe-emodin dose-dependently inhibited iNOS mRNA expression and nitric oxide (NO) production at 5-40 $\mu \mathrm{M}$. In addition, the levels of cyclooxygenase-2 (COX-2) mRNA and prostaglandin E2 $\left(\mathrm{PEG}_{2}\right)$ production were suppressed by $40 \mu \mathrm{M}$ aloe-emodin. This result indicated that aloeemodin was an effective inhibitor for these two targets. Jayaraman et al. [25] have proved that naringenin decreased the expression of iNOS and COX-2 in the liver of ethanol fed rats. Moreover, Park et al. [26] investigated the inhibiting mechanism of naringenin at the molecular level, finding that naringenin inhibited iNOS and COX-2 mRNA expression and reduced the production of $\mathrm{NO}$ and $\mathrm{PGE}_{2}$. Chao et al. [27] considered that naringenin had a stronger inhibitory effect toward iNOS and COX-2 than did an equal concentration of vitamin C. Another study showed the possible interaction between naringenin and XO. Naoghare et al. [28] proposed that $\mathrm{XO}$ was also inhibited by naringenin because the concomitant hydrophilicity and hydrophobicity within the naringenin molecule helped it to bind the active site of $\mathrm{XO}$ more strongly, thereby reducing the activity of XO. In the present study, the pharmacological network built showed 


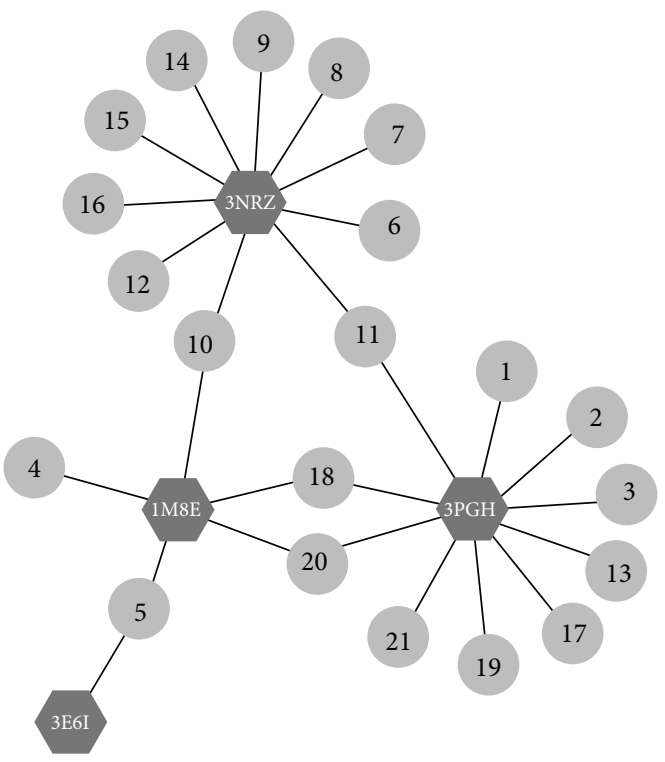

(a)

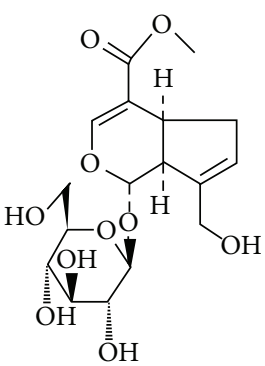

1 Geniposide

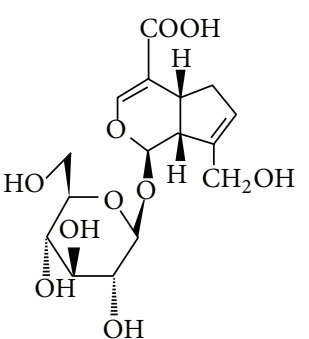

2 Geniposidic acid

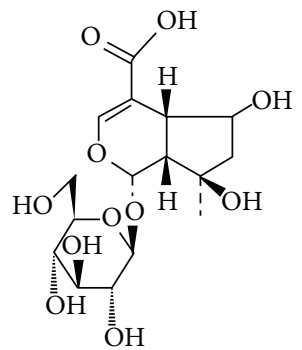

Shanzhiside<smiles>O=c1ccc2cc3ccoc3c(O)c2o1</smiles>

4 Xanthotoxol<smiles>O=c1ccc2ccc(O)cc2o1</smiles>

5 Umbelliferone<smiles>[R20]c1cc(O)c2c(c1)OC(c1ccc(O)c([R10])c1)CC2=O</smiles>

\begin{tabular}{lccl}
\multicolumn{1}{c}{$\mathrm{R}$} & $\mathrm{R}_{1}$ & $\mathrm{R}_{2}$ & Compound \\
6 Rutinose & $\mathrm{OH}$ & $\mathrm{H}$ & Eriocitrin \\
7 Neohesperidose & $\mathrm{OH}$ & $\mathrm{H}$ & Neoeriocitrin \\
8 Neohesperidose & $\mathrm{H}$ & $\mathrm{H}$ & Naringin \\
9 Rutinose & $\mathrm{OH}$ & $\mathrm{CH}_{3}$ & Hesperidin \\
$\mathbf{1 0 ~ H}$ & $\mathrm{H}$ & $\mathrm{H}$ & Naringenin \\
$\mathbf{1 1 ~ H}$ & $\mathrm{OH}$ & $\mathrm{CH}_{3}$ & Hesperetin \\
$\mathbf{1 2}$ Neohesperidose & $\mathrm{H}$ & $\mathrm{CH}_{3}$ & Poncirin
\end{tabular}

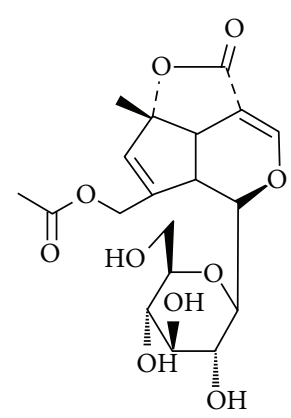

13 Asperuloside<smiles>[R]c1cc(-c2cc(=O)c3c(O)c([R])c([R20])cc3o2)ccc1OC([R])O</smiles>

$$
\begin{array}{lcccc}
\mathrm{R} & \mathrm{R}_{1} & \mathrm{R}_{2} & \mathrm{R}_{3} & \text { Compound } \\
\text { Rutinose } & \mathrm{H} & \mathrm{H} & \mathrm{H} & \text { Isorhoifolin } \\
\text { Neohesperidose } & \mathrm{H} & \mathrm{H} & \mathrm{H} & \text { Rhoifolin } \\
\text { Rutinose } & \mathrm{OH} & \mathrm{CH}_{3} & \mathrm{H} & \text { Diosmin } \\
\mathrm{H} & \mathrm{CH}_{3} \mathrm{O} & \mathrm{CH}_{3} & \mathrm{CH}_{3} \mathrm{O} & \text { Eupatilin }
\end{array}
$$

(b)

$\mathrm{R}$
14 Rutinose
15 Neohesperidose
16 Rutinose
$17 \mathrm{H}$




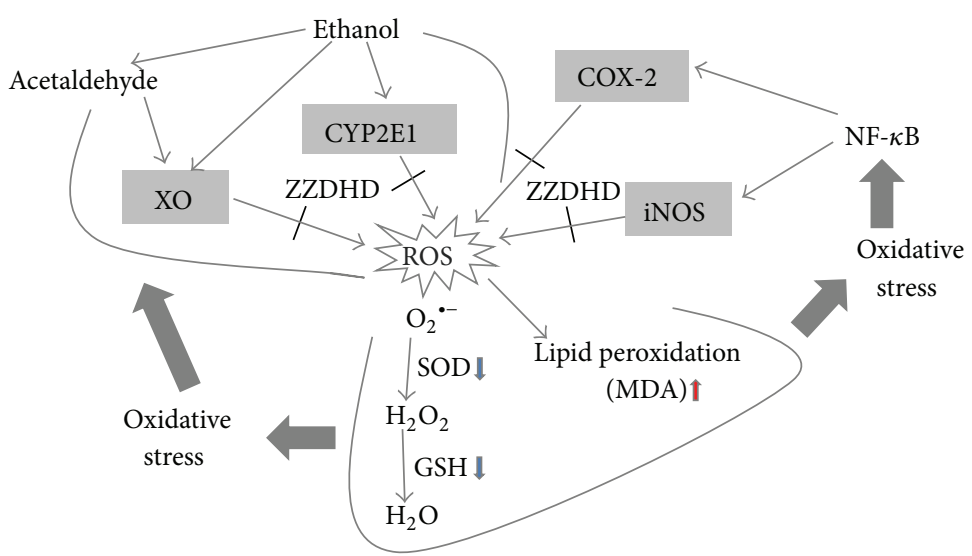

CYP2E1: PDB ID code 3E6I

COX-2: PDB ID code 3PGH
XO: PDB ID code 3NRZ

iNOS: PDB ID code $1 \mathrm{M} 8 \mathrm{E}$

FIGURE 2: Schematic illustrating proposed action mechanism by which ZZDHD prevents ALD. That ethanol induces CPY2E1, and XO causes oxidative stress in liver, due to overproduction of ROS, antioxidants reduction, and lipid peroxidation. These results, in turn, improve NF- $\kappa \mathrm{B}$ activation and then increase the expression such as in COX-2 and iNOS, further aggravating oxidative injury. Multiple components from ZZDHD (e.g., aloe-emodin and naringenin) inhibit the activation of CYP2E1, XO, COX-2, and iNOS, thereby protecting liver against the damage.

that naringenin interacted with iNOS and XO. In fact, COX2 also exhibits interactions with naringenin, but as the rank was not in the top 10, the interaction was not shown in our network.

Although the working mechanism was described from molecular point of view, it had a strong theoretical and scientific implication. Partial predicted results have been reported in some literatures, serving as a good reference value for verifying our network. Moreover, we first reported that XO had interactions with eriocitrin, neoeriocitrin, isorhoifolin, and poncirin. The antioxidant activities of eriocitrin, neoeriocitrin, isorhoifolin, and poncirin and their inhibiting effects on oxidative or ROS damages had been investigated previously [29-32], but their inhibitory effects on XO were little reported. Hence, this screening method also provided important theoretical guidance for exploring $\mathrm{XO}$ inhibitors from TCM. Drug-target network indicated the molecular mechanism of action of ZZDHD with regard to antioxidant effects. In our future studies, the details and experimental verification for novel screened active compounds will be elucidated.

3.2. Antioxidative Molecular Mechanism of ZZDHD. The antioxidation mechanisms of ZZDHD were predicted using network pharmacology. Much research has revealed that CYP2E1 plays a role in ethanol-induced liver steatosis. Ethanol initially induces CYP2E1 activation followed by increases in oxidative stress in the liver [33], and the ROS generated by CYP2E1 promotes oxidative stress. ROS leads to the production of reactive aldehydes, also with potent proinflammatory properties [8]. Oxidative stress increases NF- $\kappa \mathrm{B}$ activation, thus enhancing the expression of COX-2 and iNOS [34]. The upregulation of COX-2 expression causes the enhanced production of $\mathrm{PGE}_{2}$, which functions through its receptors $\left(\mathrm{EP}_{2}\right.$ and/or $\left.\mathrm{EP}_{4}\right)$ in hepatocytes to increase the accumulation of triglycerides [11], with further development in fatty liver. Recent studies have also shown that specific iNOS inhibitors or iNOS knockout protects against ethanolcaused oxidative stress $[35,36]$. Other sources of prooxidant agent include acetaldehyde, an excellent substrate for the enzyme $\mathrm{XO}$, which generates more toxic oxygen radicals during its oxidative catalysis [37]. Indeed, the inhibition of XO decreases ethanol- and acetaldehyde-induced lipid peroxidation.

Overproduction of ROS disrupts the balance between the oxidation and antioxidation systems, further causing oxidative stress [7]. Radical scavenging system is the body's first line of defense against oxidation, and SOD and GSH are the main components of the antioxidant protection system. SOD is a basic antioxidant enzyme responsible for catalyzing the dismutation of superoxide anion radical $\left(\mathrm{O}_{2}{ }^{-\bullet}\right)$, and its activity indirectly indicates the body's ability of scavenging free radical [38]. GSH is a major nonprotein thiol and plays a central role in coordinating the antioxidant defense process [39]. Depletion of GSH may be associated with aggravation of oxidative damage, which usually reflects the level of the organism's antioxidant ability. MDA, an end-product of lipid peroxidation, has been widely used as an indicator for the degree of lipid peroxidation [40] and it also indirectly incarnates the formation of free radicals and the degree of cell damage. Previous studies had reported that ZZDHD markedly improved SOD, GSH, and MDA, which indicated ZZDHD had contributed to the elimination of excessive oxygen free radicals and the reduction of lipid peroxidation [13].

ZZDHD modulated the activity of ethanol-metabolizing enzymes such as CYP2E1 and XO and inhibited the expression of enzymes such as COX-2 and iNOS. The above result showed that the antioxidant effect of ZZDHD may be an important influence factor for relieving oxidative stress as well as reducing the generation of lipid peroxidation induced by ethanol (Figure 2). 


\section{Conclusion}

In this paper, a drug-target network was constructed through molecular docking and network analysis. The network predicted the underlying antioxidant mechanism of ZZDHD as an effective therapeutic approach to treat alcoholic liver disease. This study demonstrated that a network pharmacologybased approach was useful for elucidating the interrelationship between complex diseases such as ALD and TCM formula interventions. Therefore, network pharmacology is a forceful tool for exploring the potential mechanism of action of TCM formula and new active ingredients. As further steps, experimental verification of the potential effective compounds after candidate screening is needed to validate the interactions between drugs and proteins based on theoretical predictions.

\section{Conflict of Interests}

The authors declare no competing financial interests.

\section{Acknowledgment}

The study was supported by the National Natural Science Foundation of China (Grant no. 81274063).

\section{References}

[1] A. Cruz and J. Muntane, "Alcoholic liver disease, oxidative stress, and antioxidants," Revista Espanola de Enfermedades Digestivas, vol. 103, no. 8, pp. 393-395, 2011.

[2] B. Gao and R. Bataller, "Alcoholic liver disease: pathogenesis and new therapeutic targets," Gastroenterology, vol. 141, no. 5, pp. 1572-1585, 2011.

[3] D. Wu and A. I. Cederbaum, "Alcohol, oxidative stress, and free radical damage," Alcohol Research and Health, vol. 27, no. 4, pp. 277-284, 2003.

[4] X. Lv, Z. Chen, J. Li et al., "Caffeine protects against alcoholic liver injury by attenuating inflammatory response and oxidative stress," Inflammation Research, vol. 59, no. 8, pp. 635-645, 2010.

[5] I. Kurose, H. Higuchi, S. Kato et al., "Oxidative stress on mitochondria and cell membrane of cultured rat hepatocytes and perfused liver exposed to ethanol," Gastroenterology, vol. 112, no. 4, pp. 1331-1343, 1997.

[6] R. M. Adibhatla and J. F. Hatcher, "Phospholipase A 2, reactive oxygen species, and lipid peroxidation in cerebral ischemia," Free Radical Biology and Medicine, vol. 40, no. 3, pp. 376-387, 2006.

[7] E. Albano, "Alcohol, oxidative stress and free radical damage," Proceedings of the Nutrition Society, vol. 65, no. 3, pp. 278-290, 2006.

[8] H. Tilg, A. R. Moschen, and N. C. Kaneider, "Pathways of liver injury in alcoholic liver disease," Journal of Hepatology, vol. 55, no. 5, pp. 1159-1161, 2011.

[9] S. Kato, T. Kawase, J. Alderman, N. Inatomi, and C. S. Lieber, "Role of xanthine oxidase in ethanol-induced lipid peroxidation in rats," Gastroenterology, vol. 98, no. 1, pp. 203-210, 1990.

[10] X.-S. Deng and R. A. Deitrich, "Ethanol metabolism and effects: nitric oxide and its interaction," Current Clinical Pharmacology, vol. 2, no. 2, pp. 145-153, 2007.
[11] N. Enomoto, K. Ikejima, S. Yamashina et al., "Kupffer cellderived prostaglandin E2 is involved in alcohol-induced fat accumulation in rat liver," The American Journal of PhysiologyGastrointestinal and Liver Physiology, vol. 279, no. 1, pp. G100G106, 2000.

[12] J. C. Chen, "Hypothesis and clinical study of modular formulology," Journal of Chinese Medicine, vol. 12, pp. 69-80, 2001.

[13] H. Wang, F. Feng, B.-Y. Zhuang, and Y. Sun, "Evaluation of hepatoprotective effect of Zhi-Zi-Da-Huang decoction and its two fractions against acute alcohol-induced liver injury in rats," Journal of Ethnopharmacology, vol. 126, no. 2, pp. 273-279, 2009.

[14] H. Wang and F. Feng, "Identification of components in Zhi$\mathrm{Zi}-\mathrm{Da}-\mathrm{Huang}$ decoction by HPLC coupled with electrospray ionization tandem mass spectrometry, photodiode array and fluorescence detectors," Journal of Pharmaceutical and Biomedical Analysis, vol. 49, no. 5, pp. 1157-1165, 2009.

[15] S. Li, T. P. Fan, W. Jia, A. Lu, and W. Zhang, "Network pharmacology in traditional Chinese medicine," Evidence-Based Complementary and Alternative Medicine, vol. 2014, Article ID 138460, 2 pages, 2014.

[16] W. Tao, X. Xu, X. Wang et al., "Network pharmacology-based prediction of the active ingredients and potential targets of Chinese herbal Radix Curcumae formula for application to cardiovascular disease," Journal of Ethnopharmacology, vol. 145, no. 1, pp. 1-10, 2013.

[17] J. Gu, H. Zhang, L. Chen, S. Xu, G. Yuan, and X. Xu, "Drugtarget network and polypharmacology studies of a Traditional Chinese Medicine for type II diabetes mellitus," Computational Biology and Chemistry, vol. 35, no. 5, pp. 293-297, 2011.

[18] X. Liang, H. Li, and S. Li, "A novel network pharmacology approach to analyse traditional herbal formulae: the Liu-WeiDi-Huang pill as a case study," Molecular BioSystems, vol. 10, no. 5, pp. 1014-1022, 2014.

[19] H. Li, L. Zhao, B. Zhang et al., "A network pharmacology approach to determine active compounds and action mechanisms of ge-gen- qin-lian decoction for treatment of type 2 diabetes," Evidence-Based Complementary and Alternative Medicine, vol. 2014, Article ID 495840, 12 pages, 2014.

[20] J. Zhao, P. Jiang, and W. Zhang, "Molecular networks for the study of TCM pharmacology," Briefings in Bioinformatics, vol. 11, no. 4, pp. 417-430, 2009.

[21] I. S. Young and J. V. Woodside, "Antioxidants in health and disease," Journal of Clinical Pathology, vol. 54, no. 3, pp. 176-186, 2001.

[22] Z.-M. Lu, W.-Y. Tao, X.-L. Zou, H.-Z. Fu, and Z.-H. Ao, "Protective effects of mycelia of Antrodia camphorata and Armillariella tabescens in submerged culture against ethanol-induced hepatic toxicity in rats," Journal of Ethnopharmacology, vol. 110, no. 1, pp. 160-164, 2007.

[23] S.-L. Liu, C.-C. Cheng, C.-C. Chang et al., "Discovery of serum biomarkers of alcoholic fatty liver in a rodent model: C-reactive protein," Journal of Biomedical Science, vol. 18, no. 1, article 52, 2011.

[24] M.-Y. Park, H.-J. Kwon, and M.-K. Sung, "Evaluation of aloin and aloe-emodin as anti-inflammatory agents in aloe by using murine macrophages," Bioscience, Biotechnology and Biochemistry, vol. 73, no. 4, pp. 828-832, 2009.

[25] J. Jayaraman, V. A. S. Jesudoss, V. P. Menon, and N. Namasivayam, "Anti-inflammatory role of naringenin in rats with ethanol induced liver injury," Toxicology Mechanisms and Methods, vol. 22, no. 7, pp. 568-576, 2012. 
[26] H. Y. Park, G.-Y. Kim, and Y. H. Choi, "Naringenin attenuates the release of pro-inflammatory mediators from lipopolysaccharide-stimulated BV2 microglia by inactivating nuclear factor- $\kappa \mathrm{B}$ and inhibiting mitogen-activated protein kinases," International Journal of Molecular Medicine, vol. 30, no. 1, pp. 204-210, 2012.

[27] C.-L. Chao, C.-S. Weng, N.-C. Chang, J.-S. Lin, S.-T. Kao, and F.-M. Ho, "Naringenin more effectively inhibits inducible nitric oxide synthase and cyclooxygenase-2 expression in macrophages than in microglia," Nutrition Research, vol. 30, no. 12, pp. 858-864, 2010.

[28] P. K. Naoghare, H. T. Kwon, and J. M. Song, "On-chip assay for determining the inhibitory effects and modes of action of drugs against xanthine oxidase," Journal of Pharmaceutical and Biomedical Analysis, vol. 51, no. 1, pp. 1-6, 2010.

[29] D. N. Olennikov, N. K. Chirikova, Z. M. Okhlopkova, and I. S. Zulfugarov, "Chemical composition and antioxidant activity of Tánara Ótó (Dracocephalum palmatum Stephan), a medicinal plant used by the North-Yakutian nomads," Molecules, vol. 18, no. 11, pp. 14105-14121, 2013.

[30] S. Tan, M. Li, X. Ding et al., "Effects of Fortunella margarita fruit extract on metabolic disorders in high-fat diet-induced obese C57BL/6 mice," PLoS ONE, vol. 9, no. 4, Article ID e93510, 2014.

[31] K.-I. Minato, Y. Miyake, S. Fukumoto et al., "Lemon flavonoid, eriocitrin, suppresses exercise-induced oxidative damage in rat liver," Life Sciences, vol. 72, no. 14, pp. 1609-1616, 2003.

[32] A. C. E. Graziano, V. Cardile, L. Crascì et al., "Protective effects of an extract from Citrus bergamia against inflammatory injury in interferon-gamma and histamine exposed human keratinocytes," Life Sciences, vol. 90, no. 25-26, pp. 968-974, 2012.

[33] L. Yang, D. Wu, X. Wang, and A. I. Cederbaum, "Cytochrome P4502E1, oxidative stress, JNK, and autophagy in acute alcoholinduced fatty liver," Free Radical Biology and Medicine, vol. 53, no. 5, pp. 1170-1180, 2012.

[34] M. Tahir, M. U. Rehman, A. Lateef et al., "Diosmin protects against ethanol-induced hepatic injury via alleviation of inflammation and regulation of TNF- $\alpha$ and NF- $\kappa$ B activation," Alcohol, vol. 47, no. 2, pp. 131-139, 2013.

[35] A. A. Nanji, S. S. Greenberg, S. R. Tahan et al., "Nitric oxide production in experimental alcoholic liver disease in the rat: role in protection from injury," Gastroenterology, vol. 109, no. 3, pp. 899-907, 1995.

[36] S. E. McKim, E. Gäbele, F. Isayama et al., "Inducible nitric oxide synthase is required in alcohol-induced liver injury: studies with knockout mice," Gastroenterology, vol. 125, no. 6, pp. 1834$1844,2003$.

[37] C. Guerri, C. Montoliu, and J. Renau-Piqueras, "Involvement of free radical mechanism in the toxic effects of alcohol: implications for fetal alcohol syndrome," Advances in Experimental Medicine and Biology, vol. 366, pp. 291-305, 1994.

[38] J. M. Matés, "Effects of antioxidant enzymes in the molecular control of reactive oxygen species toxicology," Toxicology, vol. 153, no. 1-3, pp. 83-104, 2000.

[39] V. Damodara Reddy, P. Padmavathi, and N. C. Varadacharyulu, "Emblica officinalis protects against alcohol-induced liver mitochondrial dysfunction in rats," Journal of Medicinal Food, vol. 12, no. 2, pp. 327-333, 2009.

[40] M. Zhao, Y.-Q. Du, L. Yuan, and N.-N. Wang, "Protective effect of puerarin on acute alcoholic liver injury," The American Journal of Chinese Medicine, vol. 38, no. 2, pp. 241-249, 2010. 


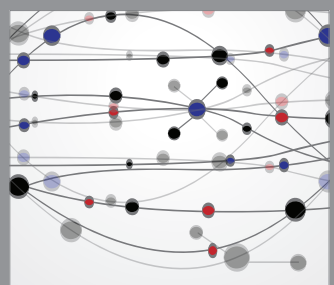

The Scientific World Journal
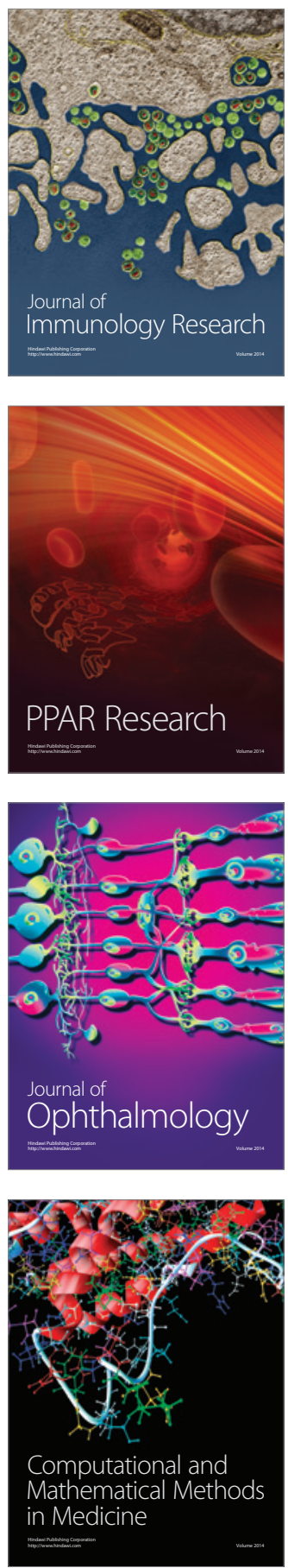

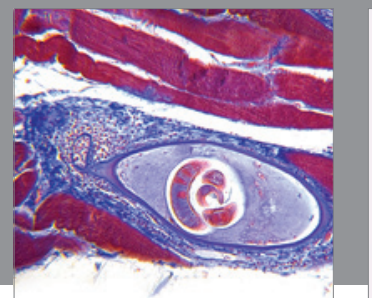

Gastroenterology

Research and Practice
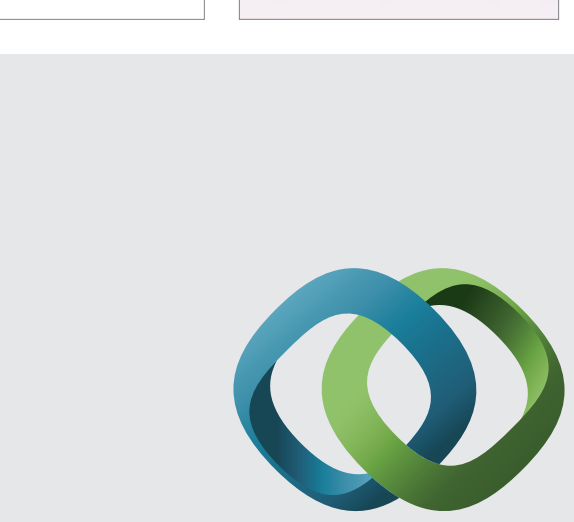

\section{Hindawi}

Submit your manuscripts at

http://www.hindawi.com
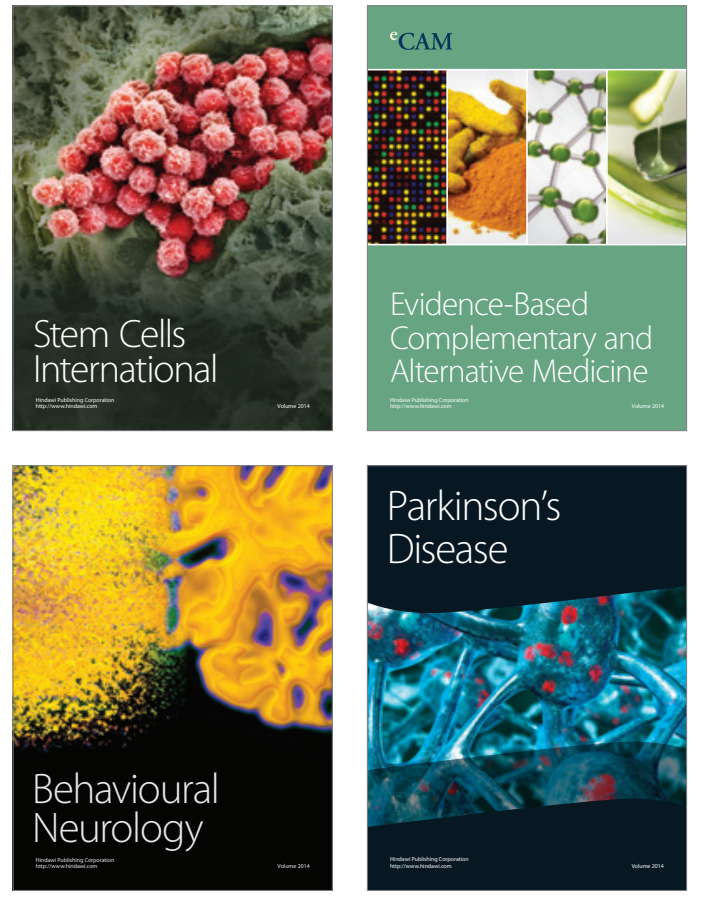
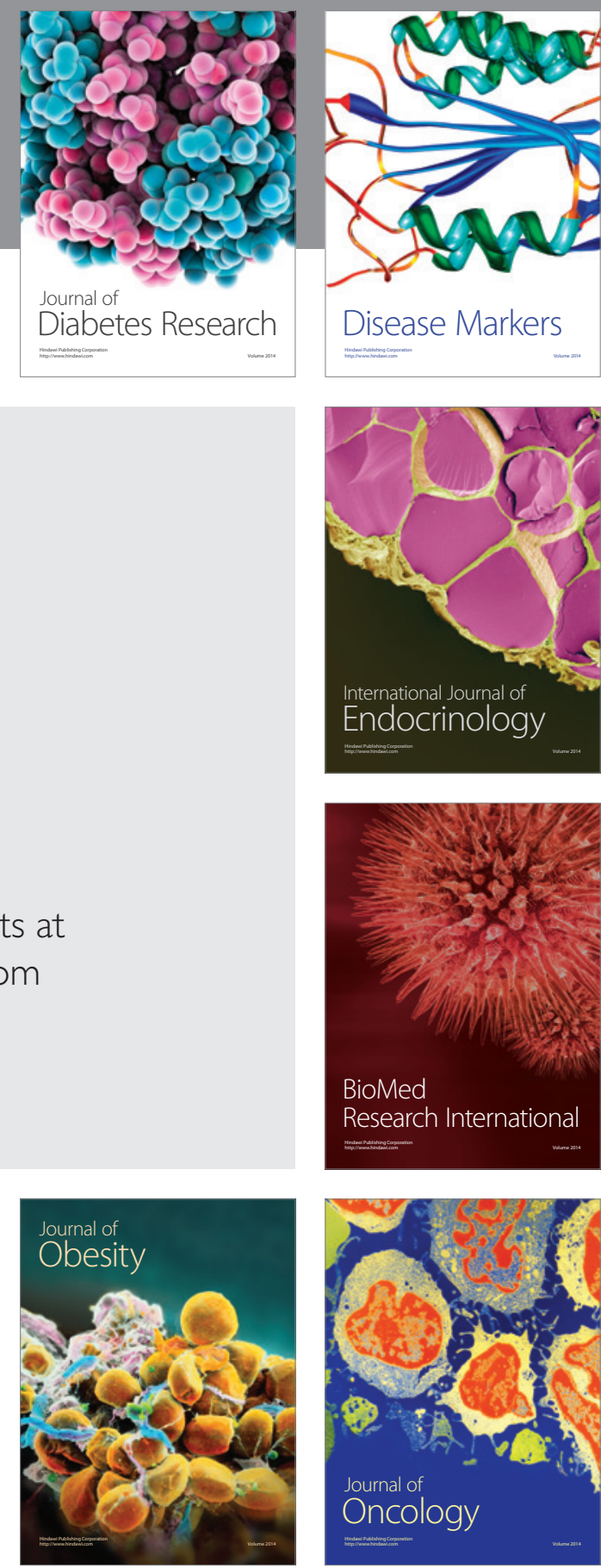

Disease Markers
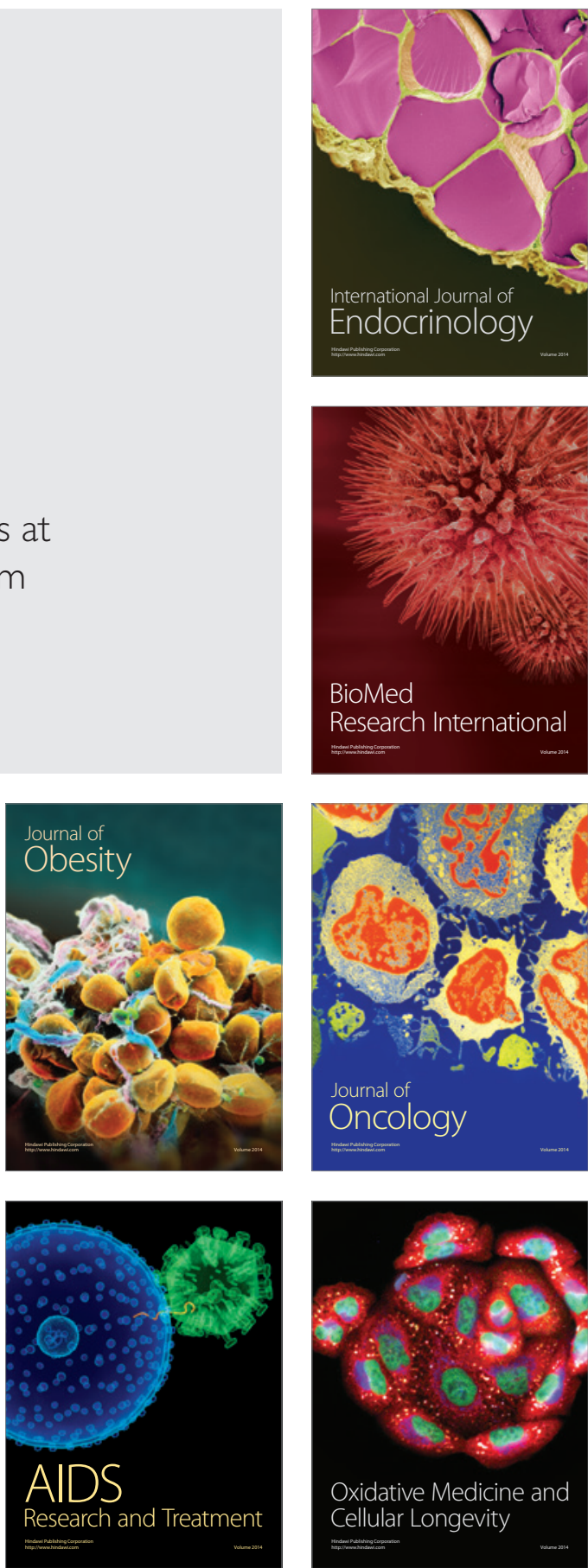